\title{
Role of Non-metallic Inclusions and Twins on the Variability in Fatigue Life in Alloy 718 Nickel Base Superalloy
}

\author{
Damien Texier, Jean-Charles Stinville, Marie-Agathe Charpagne, \\ Zhe Chen, Valery Valle, Patrick Villechaise, Tresa M. Pollock, \\ and Jonathan Cormier
}

\begin{abstract}
Non-metallic inclusions (NMIs) and slip bands parallel to and slightly offset from twin boundaries are observed to be preferential sites for fatigue crack nucleation in wrought superalloys. Potential interactions between NMI cracking and slip activity within neighboring grains or at twin boundaries were investigated under monotonic tensile loading (up to $1.3 \%$ total strain) at room temperature. High resolution- and Heaviside-digital image correlation measurements were performed during interrupted tensile loading to identify strain localization, associated slip systems, and damage initiation. Different mechanisms and scenarios were identified: (1) Microplasticity generally starts at twin boundaries even at stresses as low as $70 \%$ of the macroscopic yield strength, (2) transgranular slip activity intensively develops above the macroscopic yield stress, (3) intense slip activity develops near and parallel to $21 \%$ of the twin boundaries intercepting NMIs, (4) $7 \%$ of the twin boundaries intercepting NMIs lead to slip-assisted NMI cracking, (5) no transgranular slip activity participates in NMI cracking, (6) the fraction of cracked NMIs progressively increases with the load, and (7) within the NMIs that initiated cracks, $67 \%$ cracked below $90 \%$ of the macroscopic yield strength without the presence of slip activity in the neighboring grains. While slip-assisted NMI cracking was evidenced in the present study, most NMI cracking is due to strain incompatibility between NMIs and neighboring
\end{abstract}

D. Texier $(\square)$

Institut Clement Ader (ICA)-UMR CNRS 5312, Université de Toulouse, CNRS, INSA, UPS, Mines Albi, ISAE-SUPAERO, Campus Jarlard, 81013 Albi Cedex 09, France

e-mail: damien.texier@mines-albi.fr

J.-C. Stinville - M.-A. Charpagne - Z. Chen - T. M. Pollock Materials Department, University of California Santa Barbara, Santa Barbara, CA 93106, USA

V. Valle $\cdot$ P. Villechaise $\cdot$ J. Cormier

Institut Pprime, UPR CNRS 3346, ISAE-ENSMA, 1 Avenue

Clément Ader, BP 4010986961 Futuroscope-Chasseneuil, France grains at the high end of the elastic regime without slip interaction.

\section{Keywords}

Non-metallic inclusions (NMIs) • Twin boundary • Fatigue - In-situ tensile testing • High resolution-digital image correlation (HR-DIC)

\section{Introduction}

The direct aged version of the Alloy 718 nickel base superalloy (718DA) exhibits improved tensile (yield strength) and fatigue properties (average fatigue life) up to intermediate temperatures in comparison with the solution treated variant of the alloy [1-3]. Such improvements are attributed to a smaller grain size and a higher amount of strengthening $\gamma^{\prime \prime}-\mathrm{Ni}_{3} \mathrm{Nb}$ precipitates, and not due to residual forging plastic strains [3]. This provides improvement in the average fatigue lifetime of Alloy 718 structural components in the aerospace industries such as turbine disks for aero-engines. However, this improvement in fatigue life is at the cost of higher variability on fatigue life in the low, high, and very high cycle fatigue regimes [4]. This significant variability is illustrated in Fig. 1a in the low cycle fatigue (LCF) regime under tension-tension loading at intermediate temperature $\left(R_{\varepsilon}=0.05\right.$ and $\left.f=1 \mathrm{~Hz}\right)$ [5]. Such high variability is associated with crack initiation processes from non-metallic inclusions (NMIs) such as niobium-rich carbides (NbC), titanium-rich nitrides (TiN), and carbo-nitrides [5-7]. Alloy 718 is particularly prone to crack initiation from NMIs under fatigue loading for grain sizes smaller or at a similar scale to the NMIs at the upper end of the tail of the size distribution [7-9]. While surface and subsurface NMIs are responsible for a ten- to hundredfold debit in fatigue life, the related mechanism of crack initiation and short crack propagation is not fully understood. 
LCF tension - tension $\left(R_{\varepsilon}=0.05\right)$ at $450^{\circ} \mathrm{C} / 1 \mathrm{~Hz}$

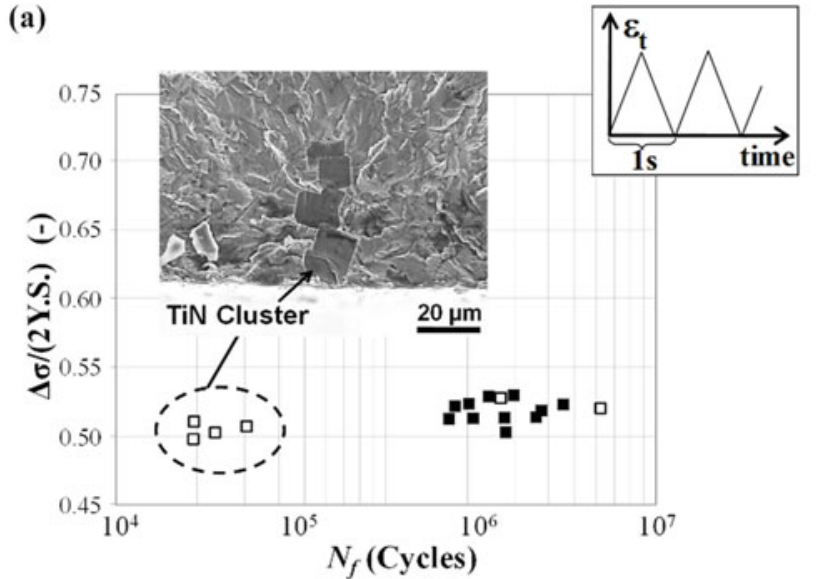

Fig. 1 Influence of microstructural features on the fatigue life of the 718DA showing abnormally low lives and associated crack initiation site: a low cycle fatigue (LCF) in tension-tension at intermediate

In parallel, annealing twin boundaries (TB) were also found as potential crack initiation sites in nickel base superalloys, including Alloy 718, due to intense strain localization that develops near and parallel to them $[6,10$ 15]. Slip activity at TB was attributed to the high elastic anisotropy of nickel base superalloys ("typical" Zener anisotropy ratio of 2.0 for 718 measured by Haldipur [16]) that promotes easy dislocation glide near and parallel to the $\{111\} \Sigma 3$ twin boundary plane. It is worth mentioning that TBs are particularly sensitive to crack initiation under fully reversed cyclic loading resulting in a large debit in fatigue life [12, 17]. Interestingly, crack initiation from TBs was found to control the fatigue life even in the presence of NMIs under certain fatigue conditions $\left(R_{\varepsilon}=-1\right.$ and $f=20 \mathrm{kHz}$ ) [6]. This was observed for a 718DA microstructure having a particularly low $\delta$-phase content and a high "TB length/surface area" ratio, as depicted in Fig. 1b. Low $\delta$-phase content is accompanied by higher $\gamma^{\prime}$ content in 718DA, i.e., a material highly sensitive to strain localization by intense slip/planar deformation.

It is therefore apparent that both NMIs and TB can affect the fatigue damage initiation processes that are at the origin of the high variability in the 718DA. Such high variability may restrict the efficient usage of the 718DA alloy for critical structural components from a design point of view, despite the overall improvement in mechanical performance. Therefore, an in-depth understanding of the role of NMIs and their neighboring microstructure will provide critical insights on the causes of variation in fatigue life. Plasticity associated with inclusions embedded in metallic matrix (nickel or steel as a continuum medium) was investigated using finite element analyses (FEA) [18-21] and more recently FEA coupled with high resolution-digital image
VHCF tension - compression $\left(R_{\varepsilon}=-1\right)$ at $\mathrm{RT} / 20 \mathrm{kHz}$

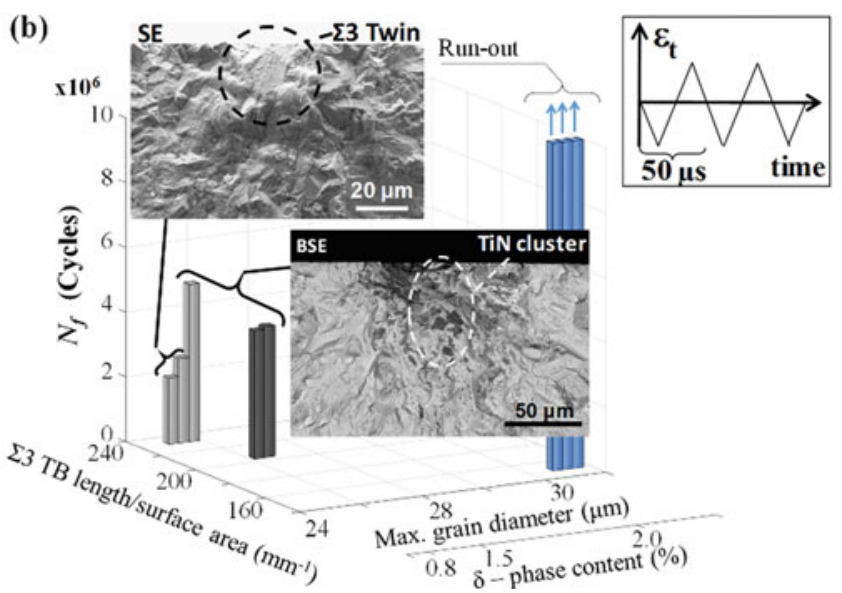

temperature [5], b very high cycle fatigue (VHCF) in tensioncompression at room temperature [6]

correlation (HR-DIC) techniques [22]. These studies demonstrated accumulation of plastic deformation at the "NMI/metallic matrix" interface under cyclic loading in the presence or not of initial damage at the NMI/matrix interface. Bandyopadhyay and Sangid used a more complex model introducing the grain structure and crystal plasticity finite element models instead of a continuum medium [23]. The model aimed at identifying competing failure modes, namely inclusion- and matrix-driven failures, depending on the inclusion stiffness, the orientation of neighboring grains, the inclusion size, NMI/matrix debonding. Twins were also considered in the numerical aggregate. The authors concluded that local plastic accumulation in the cyclically tested agglomerates results from the intricate interaction between all the above-mentioned microstructural parameters. In other words, these systematic computations and analyses suggested that small NMIs could be as detrimental as coarse NMIs, depending on the neighboring microstructure. They also confirmed via numerical simulations that surface and subsurface NMIs, i.e., close to the free-surface, are more damaging but do not systematically lead to inclusion-driven failure. Therefore, a closer analysis of strain localization and onset of damage between NMIs and their neighboring grains is needed. In addition, Kartal et al. reported the importance of the heat treatment cycle, and more particularly the last cooling step, in producing residual strain and eigenstrain at the NMI/matrix interface due to thermal expansion mismatch between NMIs and the alloy [24]. A previous experimental study [4] identified four configurations for crack initiation: (i) no propagation despite a cracked NMI, (ii) $\mathrm{NMI} /$ matrix debonding, (iii) transgranular propagation involving one slip system and slip band, and (iv) transgranular propagation involving multiple slip systems and slip 
bands. While a morphological criterion was attributed to non-propagating NMIs ("width/depth" ratio $\geq 3$ ), none of the three propagating cases were found to be more damaging than the other ones.

In this context, the present study aims to evaluate the potential interaction between NMI cracking and slip activity within neighboring grains or at twin boundaries intercepting NMIs. High resolution-digital image correlation (HR-DIC) was used to experimentally document the onset of plasticity under monotonic tensile testing, as well as the evolution of the local strain fields due to increased loading. Assessing the onset of microplasticity within the neighboring grains and damage development under monotonic tensile loading is essential to document different deformation/damage mechanisms depending on the stress-strain amplitude. The present investigation is a first step toward a better understanding of the deformation/damage mechanisms favoring short crack propagation from NMIs and twin boundaries, or from the concomitant action of both microstructural features.

\section{Experimental Procedures}

\section{Material}

Mechanical specimens were machined from wrought 718DA pancakes showing a high variability in fatigue life [6]. The microstructure consists of $13 \pm 5 \mu \mathrm{m}$ grains containing annealing twin boundaries and a $\delta$-phase content of $2.0 \%$. Grains and NMIs have similar size distribution. The nominal composition (in weight \%) of the 718DA pancakes was $\mathrm{Ni}-0.56 \mathrm{Al}-18.0 \mathrm{Cr}-17.3 \mathrm{Fe}-2.97 \mathrm{Mo}-1.0 \mathrm{Ti}-5.4(\mathrm{Nb}+\mathrm{Ta})$ $0.14 \mathrm{Co}-0.023 \mathrm{C}-0.006 \mathrm{~N}$. Specimens were radially machined from the center region of the pancakes by electro-discharge machining (EDM), with a flat dogbone-shaped geometry defined in Ref. [5]. The EDM affected layer was removed using fine-grit polishing and the specimens were mirror polished using diamond suspensions down to $1 \mu \mathrm{m}$. Specimens were then chemical-mechanically polished with $0.05 \mu \mathrm{m}$ colloidal silica for $12 \mathrm{~h}$. Such procedure enabled electron backscattered diffraction (EBSD) measurements to be performed prior to deformation.

\section{Ex-Situ and In-Situ Tensile Testing}

Ex-situ continuous monotonic tensile testing was performed using a custom $\pm 5 \mathrm{kN}$ microtensile stage under displacement control. Strain and load were continuously recorded using a microstrain gage and a $\pm 5 \mathrm{kN}$ load-cell. The stressstrain curve of the alloy is shown in Fig. 2a. In-situ tensile tests were performed in a FEI Versa3D field emission gun scanning electron microscope (FEG-SEM). Specimens were loaded in both the macroscopic elastic and plastic deformation domains, as shown with red crosses in Fig. 2 a. Macroscopic strain was measured in-situ using fiducial markers located at both ends of the gage length. The loading direction (LD) will be referred as the $x$-direction and horizontally oriented in all the micrographs and strain fields. High resolution- and Heaviside-digital image correlation (HR-DIC and H-DIC) measurements were performed at macroscopic strain of $\varepsilon_{\text {tot }}=0.45,0.61,0.70,1.0$, and $1.3 \%$ in regions that display NMIs (detailed in Sect. 2.3). Twenty regions of interest (ROIs) were tracked.

\section{High Resolution- and Heaviside-Digital Image Correlation (HR-DIC and H-DIC) Technique}

HR-DIC measurements were performed in the vicinity of NMIs to assess strain localization at the scale of elementary mechanisms of deformation and NMI cracking. Particular care was taken in applying the speckle pattern, not to affect the NMI/matrix interfaces prior to mechanical loading (detailed in Sect. 2.1). Gold nanoparticles with an average diameter of $40 \mathrm{~nm}$ were deposited at the surface of the specimen following the procedure developed by Kammers et al. [25]. The density of gold nanoparticles was about $85 \pm 10 \mu \mathrm{m}^{-2}$ to enable local gray-level contrasts compatible with the measurement of sub-grain kinematic fields using HR-DIC (see e.g., insert in Fig. 2c). Distortion errors inherent to SEM imaging were minimized using SEM parameters following the guidelines of Kammers and Daly [26] and Stinville et al. [27]. Images with a resolution of $6144 \times 4096 \mathrm{pxl}^{2}$ were acquired with a horizontal field width of $120 \mu \mathrm{m}$, i.e., a pixel size of $19.5 \mathrm{~nm}$, using a $5 \mathrm{kV}$ acceleration voltage, $0.34 \mathrm{nA}$ electron beam current, and a $20 \mu$ s dwell time. Reference images were acquired prior to monotonic deformation. Deformed images were acquired at different macroscopic strain-stress conditions under loading, illustrated with red crosses in Fig. 2a. The H-DIC method was used to accurately assess discontinuities in kinematics fields related to cracking [28] or slip activity [29]. Displacement maps were computed using a measurement grid of $3 \mathrm{pxl}(58 \mathrm{~nm})$, an initial subset size of $32 \times 32 \mathrm{pxl}^{2}$ $\left(624 \times 624 \mathrm{~nm}^{2}\right)$ and a bicubic interpolation. In-plane strain maps in $x$ - and $y$-directions $\left(\varepsilon_{x x}, \varepsilon_{y y}\right.$, and $\left.\varepsilon_{x y}\right)$ as well as discontinuity vectors (amplitude and direction) were automatically computed with the H-DIC method for slip and damage identification. The present experimental investigation does not consider the residual strain state in the grains adjacent to NMIs arising from thermal excursions during heat treatments, i.e., prior to tensile testing [23, 24]. This residual strain state could affect the onset of slip localization in grains adjacent to NMIs. 
(a)

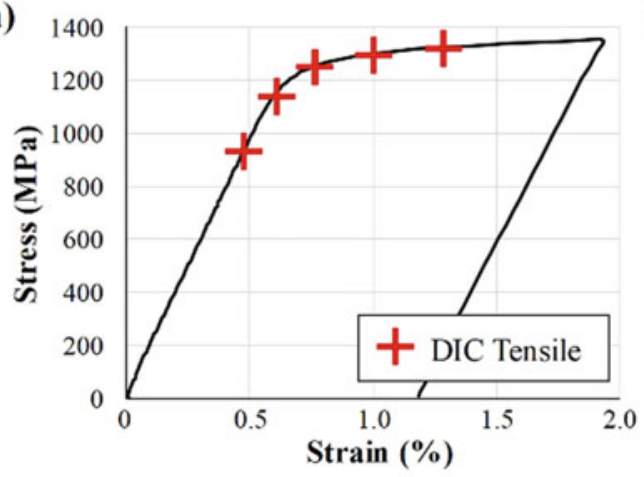

(c)

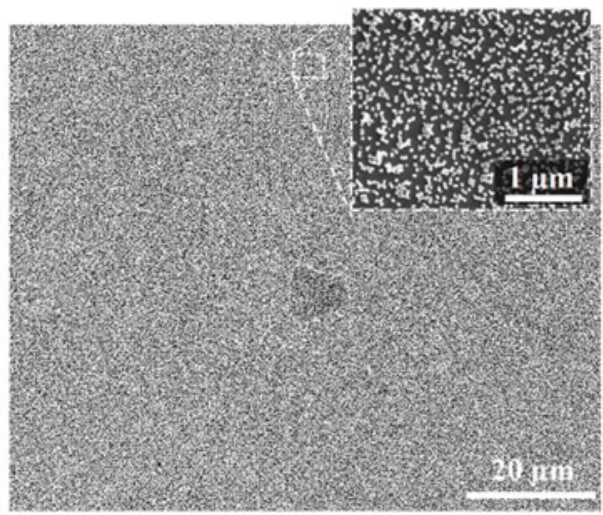

(e)

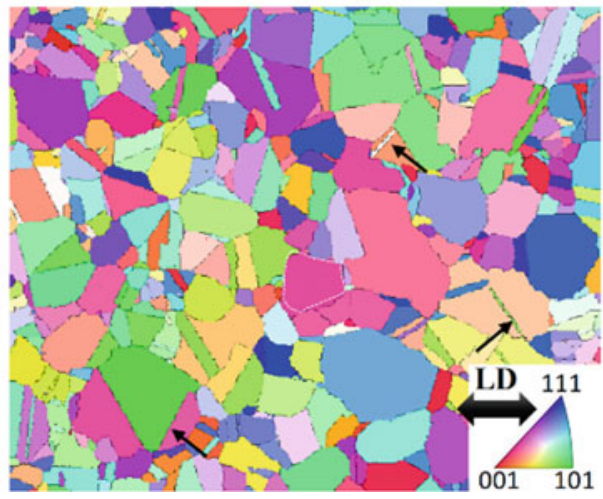

(b)

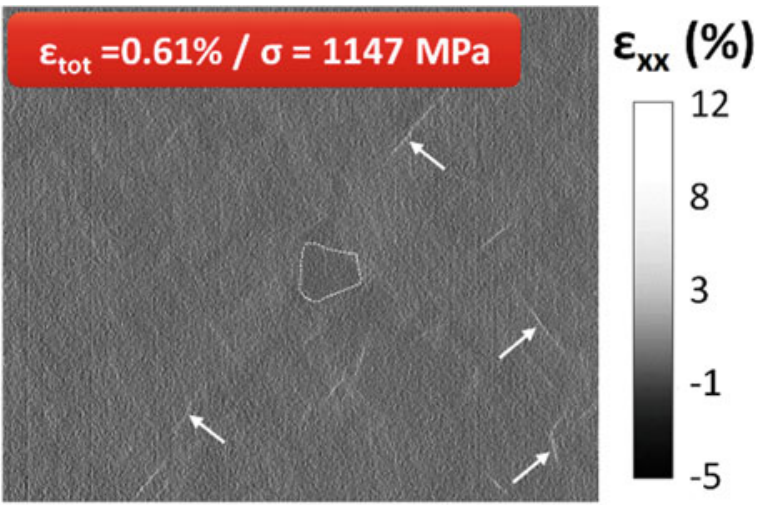

(d)

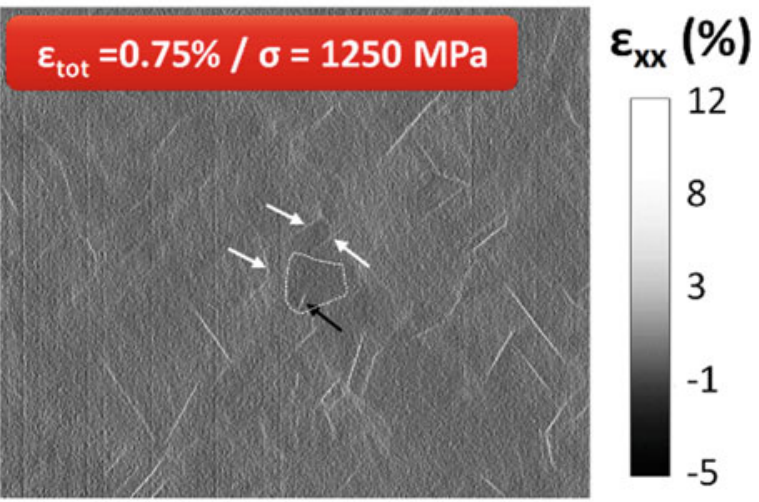

(f)

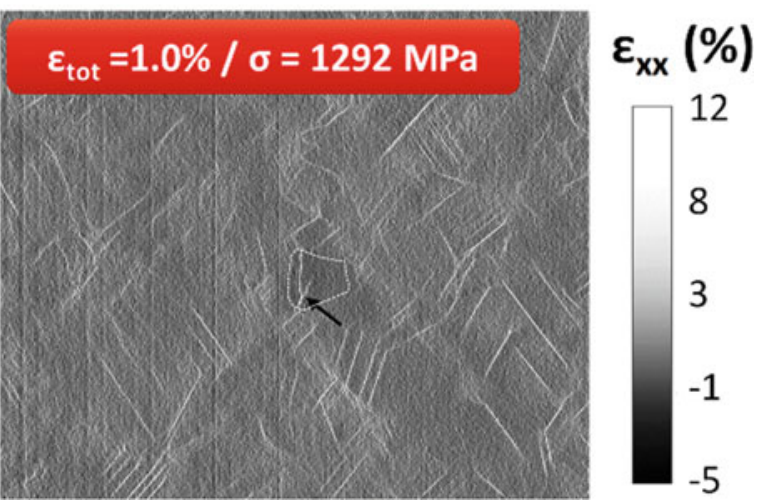

Fig. 2 Evolution of strain localization and cracking under tensile loading: a macroscopic stress-strain curve of the tested 718DA and loading steps for high resolution-digital image correlation (HR-DIC) measurements, $\mathbf{c}$ SEM micrograph used for HR-DIC in the vicinity of a nitride (TiN) inclusion showing the speckle pattern, e EBSD map of the ROI colored according the IPF colors with projection along the loading direction (LD), b, $\mathbf{d}$ and $\mathbf{f} \varepsilon_{x x}$ strain maps at different stress-strain levels showing strain localization first in the grains far from the TiN (white arrows Fig. 2b), then strain localization in the grains neighboring the TiN (white arrows in Fig. 2d) and cracking of this NMIs (black arrow in Fig. 2d, f)

\section{Electron Backscattered Diffraction (EBSD) Analyses}

The microstructure of the twenty ROIs was mapped by EBSD prior to deformation. A FEI Versa3D FEG-SEM equipped with an EDAX OIM-Hikari XM4 EBSD camera was used. Rectangular measurement grids with a step size of
$0.25 \mu \mathrm{m}$ were carried out on $100 \times 100 \mu \mathrm{m}^{2}$ areas with the NMIs centered in the analysis field. Diffraction patterns were acquired using an accelerating voltage of $20 \mathrm{kV}$, a beam current of $0.2 \mathrm{nA}$ and a binning of $4 \times 4$ of the diffraction patterns. EBSD measurements were used to assess the grain orientations and identify slip and cleavage events indicated by HR-DIC and H-DIC measurements. 


\section{Results and Discussion}

As aforementioned, twenty ROIs were investigated to document the evolution of the plastic localization at the sub-grain level in the vicinity of NMIs and in the metallic matrix under tensile loading. Different strain-stress conditions were investigated per ROI from $70 \%$ of the $0.2 \%$ offset yield strength (YS) to $0.8 \%$ macroscopic plastic strain. One hundred $\varepsilon_{x x}$ strain fields were thus analyzed to identify and classify different deformation and damage mechanisms. The proximity of the grains to the NMI will be taken into account: "grains in the vicinity of the NMI" and "grains far from the NMI" correspond to "grains directly adjacent to the NMI" and "grains at least $15 \mu \mathrm{m}$ distant from the NMI, i.e., at least distant from a grain." This distinction is justified due to the particular focus on the slip-assisted NMI cracking or not cracking. Transgranular slip activity and slip near and parallel to TB will also be dissociated. Three case studies will be presented in order to present and illustrate the different mechanisms identified in the present study.

\section{Case Study \#1: NMI Cracking Without Slip Interaction}

A case study of cracking in a TiN inclusion at an intermediate loading condition is illustrated in Fig. 2. The possibility of assessing slip events in the metallic matrix and cracking in the TiN under loading is demonstrated. A white dashed contour identifies the TiN location in $\varepsilon_{x x}$ strain maps and EBSD map for reading conveniences.

The onset of plasticity was observed along a few annealing twin boundaries far from NMIs at $90 \%$ of Y.S, i.e., $\varepsilon_{\text {tot }}=0.61 \%-\sigma=1147 \mathrm{MPa}$ (white arrows in Fig. $2 \mathrm{~b}$ corresponding to black arrows in Fig. 2e). No evidence of strain localization was found within the NMI or in its neighborhood. Interestingly, the TiN inclusion appears slightly darker than the matrix in $\varepsilon_{x x}$ strain fields, resulting from a higher stiffness regardless of the orientation of the NMIs or the grains. This latter observation is similar for all ROIs, i.e., independently of the nature of the NMIs. This difference in elastic properties is in good agreement with the literature [16, 30, 31]. Calculation of the elastic properties of the $\mathrm{TiN}$ and $\mathrm{NbC}$ inclusions and the superalloy matrix for various directions have shown that the superalloy matrix along its stiffest direction $\left(E_{[111]}=260 \mathrm{GPa}\right)$ is softer than $\mathrm{NbC}$ and TiN inclusions along their softer direction $\left(E_{[001]}=416 \mathrm{GPa}\right.$ for $\mathrm{NbC}$ and $E_{[001]}=417 \mathrm{GPa}$ for TiN $)$.

At higher stresses, close to the macroscopic yielding of the material $\left(\varepsilon_{\text {tot }}=0.75 \%-\sigma=1250 \mathrm{MPa}\right)$, more slip bands developed in the surrounding grains, even in contact with the NMI (white arrows in Fig. 2d). At this macroscopic strain level, several slip systems are activated near and parallel to TB but intragranular slip events are also observed. This dual participation of intense slip localization close to $\mathrm{TB}$ and within grains has also been reported by Liu et al. [15] in conventionally processed 718 alloy. However, strongest strain localizations remain along a few annealing twin boundaries far from NMIs. The TiN inclusion cracked in the bottom region (black arrow in Fig. 2d) but no slip event was detected within the superalloy grain in contact with the crack tip. Thus, the TiN inclusion cracking is not assisted by intense strain localization in the matrix in this case.

For stress-strain conditions in the macroscopic plastic domain, strain localization is more and more intense near TB but a greater number of slip bands developed transgranularly (Fig. 2f). This multiplicity of transgranular slip activity was confirmed by Liu et al. [15]. At this stress-strain level, the TiN inclusion fully cracked with a complex crack path. Slip events are now observed within adjacent grains on both NMI crack tips. This specific ROI is thus a typical case of damage transfer from a cracked NMI to the superalloy matrix but NMI cracking was not assisted by slip deformation in the adjacent grains.

\section{Case Study \#2: Slip-Assisted NMI Cracking with Twin Boundary Contribution}

A second case study is presented in Fig. 3 to show a different scenario of slip/cracking interaction for a carbonitride. As for the former case study, a white dashed contour was used to locate the NbCTiN in $\varepsilon_{x x}$ strain maps and EBSD map. Despite different compositions (gray contrast in Fig. 3a), NbC and TiN inclusions were found to have very similar orientations (less than $2^{\circ}$ misorientation, i.e., same color code on IPF map in Fig. 3b) due to epitaxial growth and similar elastic properties.

Initial microplasticity events were developed in the vicinity of the carbonitride at $90 \%$ of YS $\left(\varepsilon_{\text {tot }}=0.61 \%-\right.$ $=1147 \mathrm{MPa}$ ). Strain localization nearly parallel to the $\mathrm{NMI} /$ matrix interface was observed within two neighboring grains, as depicted by white arrows in Fig. 3b, c. It is not straightforward to identify in which grain the onset of plasticity started. Therefore, a detailed analysis of both grains will be presented. The two grains were denoted "GrA" and "GrB" and labeled on the EBSD map. The slip traces correspond to the $(\overline{1} 11)$ and (111) planes in GrA and $\mathrm{GrB}$, respectively.

At $\varepsilon_{\text {tot }}=0.75 \%-\sigma=1250 \mathrm{MPa}$, strain localization intensifies within the former slip bands and slip transfer to an adjacent grain occurs (white arrow in Fig. 3d). Interestingly, slip activity is also detected near and parallel to a TB (black 

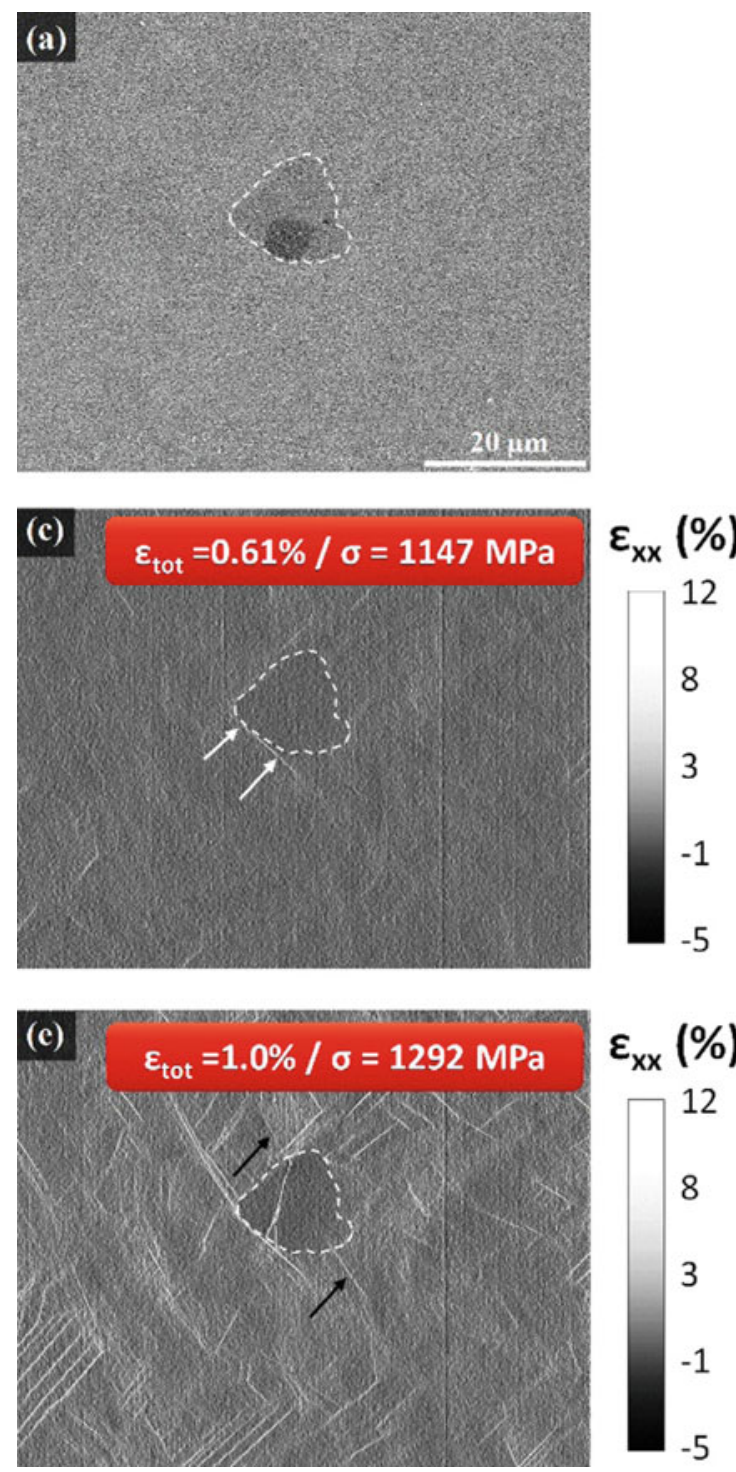

Fig. 3 Evolution of strain localization and cracking under tensile loading: a SEM micrographs used for HR-DIC showing the region of interest (ROI) in the vicinity of a carbonitride (NbCTiN), b EBSD map of the ROI represented as inverse pole figures (IFP) maps in reference

arrow in Fig. 3d). As aforementioned, other slip bands develop in other locations in the superalloy matrix. At 1.0\% total strain, the carbonitride cracked in the region where microplasticity started to develop parallel and close to the $\mathrm{NMI} /$ matrix interface. Interestingly, a slip band along a TB was observed to develop at the NMI crack tip (upper black arrow in Fig. 3b, e). At this point, it is not possible to identify whether the first event was either slip activity along the TB or NMI cracking. In addition, strain localization intensifies in the former slip bands near the TB (lower black arrow in Fig. 3e).

At $1.3 \%$ total strain, the carbonitride cracked a second time where the intense slip activity near the TB was
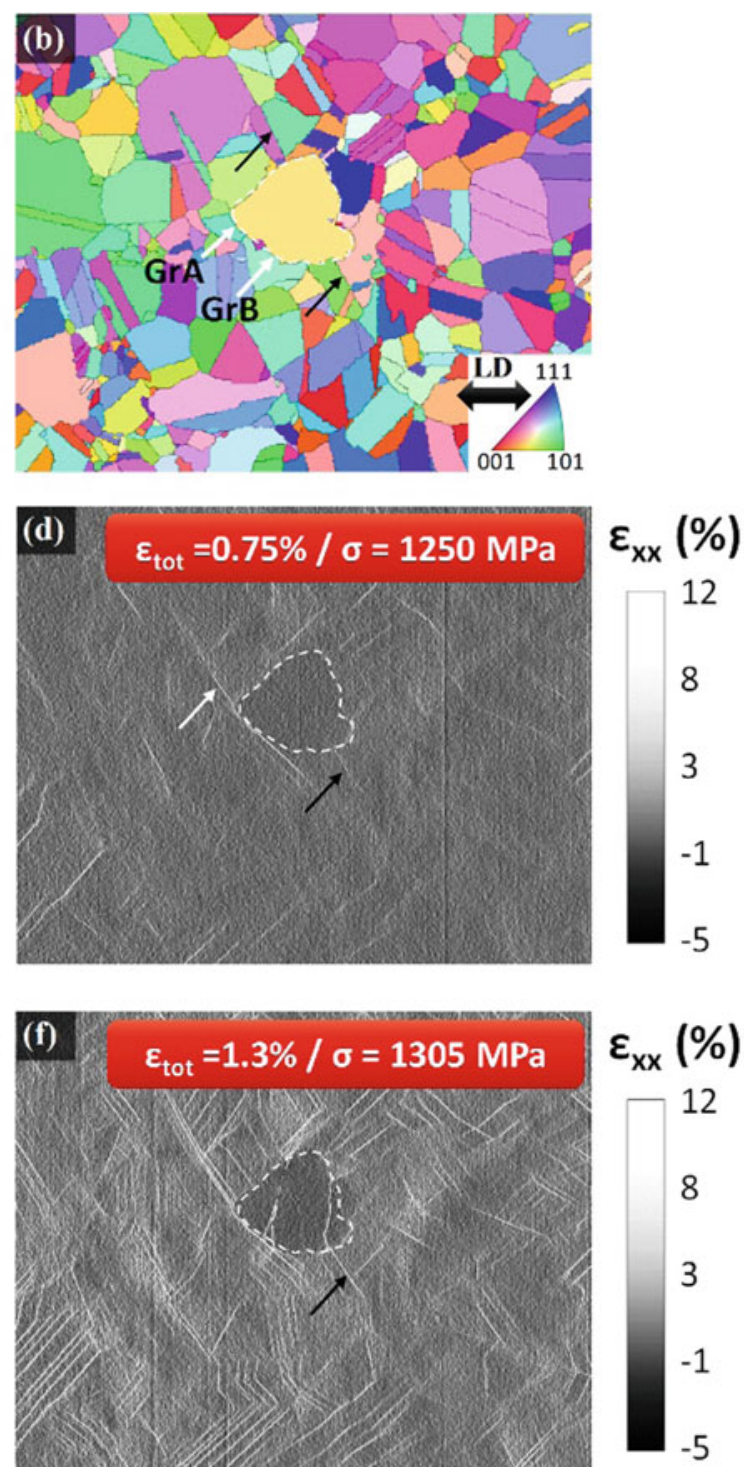

to the loading direction (LD), $\mathbf{c}-\mathbf{f} \varepsilon_{x x}$ strain maps at different stressstrain levels showing strain localization parallel to the NMI/matrix interface (white arrows in Fig. 3c), then strain localization near a TB (black arrows in Fig. 3d, e), leading to the NMI cracking (Fig. 3f)

observed. This latter cracking demonstrates the possibility of NMI cracking assisted by slip localization, and particularly close and parallel to TB due to intense plastic accumulation at the NMI/matrix interface and the brittleness of NMIs at room temperature.

\section{Case Study \#3: Slip Transfer for Two Slip Traces Parallel to the NMI/Matrix Interface}

Slip traces parallel to the $\mathrm{NMI} /$ matrix interface were observed as early plasticity events developing near the NMI in the ROI for the case study \#2 (Fig. 3c), prior to NMI 
cracking. A closer examination of such strain localization is thus needed. Strain profiles $\left(\varepsilon_{x x}\right)$ across the slip bands developing in GrA and GrB (grains labeled in Sect. 3.2) were extracted from $\varepsilon_{x x}$ maps to document slip activity with the onset of microplasticity (Fig. 4). Interestingly, strain localization is observed in GrA but not in GrB for the stress-strain conditions at $70 \%$ of Y.S $\left(\varepsilon_{\text {tot }}=0.45 \%-\sigma=\right.$ $900 \mathrm{MPa})$. As aforementioned, slip traces observed on the $\varepsilon_{x x}$ maps correspond to the $(\overline{1} 11)$ and $(1 \overline{1} 1)$ planes from GrA and GrB, respectively.

Both slip traces correspond to the $\{111\}$ planes having slip systems with the highest Schmid factor values $(\mu)$ according to the LD, among the twelve slip systems possibly active per grain. Potential slip systems matching with GrA and GrB slip traces are reported in Table 1 with $\mu$ values according to the $\mathrm{LD}$, and Burgers vector expressed in the sample coordinates. While the highest Schmid factor value is maximal for GrB, onset of slip localization started within $\mathrm{GrA}\left(\mu_{\mathrm{GrA}} \max .=\right.$ 0.433). Schmid factor values lower than 0.45 (according to the LD) are relatively low for activation of the associated slip system. This is likely due to the more complex state of stress in the polycrystalline microstructure. Plastic accumulation and damage at NMI/matrix interface under cyclic loading are already reported in the literature both in numerical [18, 20, 21, 23] and experimental [4] studies. Using HR-DIC and H-DIC, it is possible to extract from the in-plane kinematic field the displacement jump vector resulting from the shearing process at the slip band [29]. Interestingly, the displacement jump vector measured in GrA corresponded to the projection of the cumulated Burgers vector $(\overline{1} 11)[01 \overline{1}]$ with the highest $\mu$ (red column in Table 1).

At $90 \%$ of Y.S $\left(\varepsilon_{\text {tot }}=0.61 \%-\sigma=1147 \mathrm{MPa}\right)$, strain localization intensifies across GrA slip band, but the slip band across GrB produced more intense strain localization than for GrA. Similar slip system analyses of $\mathrm{GrB}$ slip band were conducted using HR-DIC and H-DIC measurements. The (111) $[\overline{101}]$ slip system was activated with a $\mu$ equal to

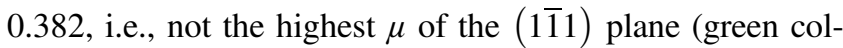
umn in Table 1). The scalar products between the Burgers vector identified in GrA and the three possible Burgers vectors in $\mathrm{GrB}$ were calculated and reported in Table 1. Interestingly, the Burgers vector of the active slip system in GrB, i.e. $(1 \overline{1} 1)[\overline{101}]$, was less misoriented from the initial slip (a)

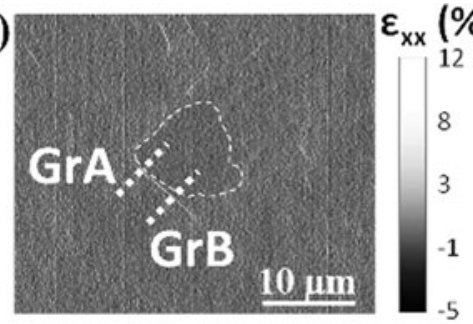

(b)

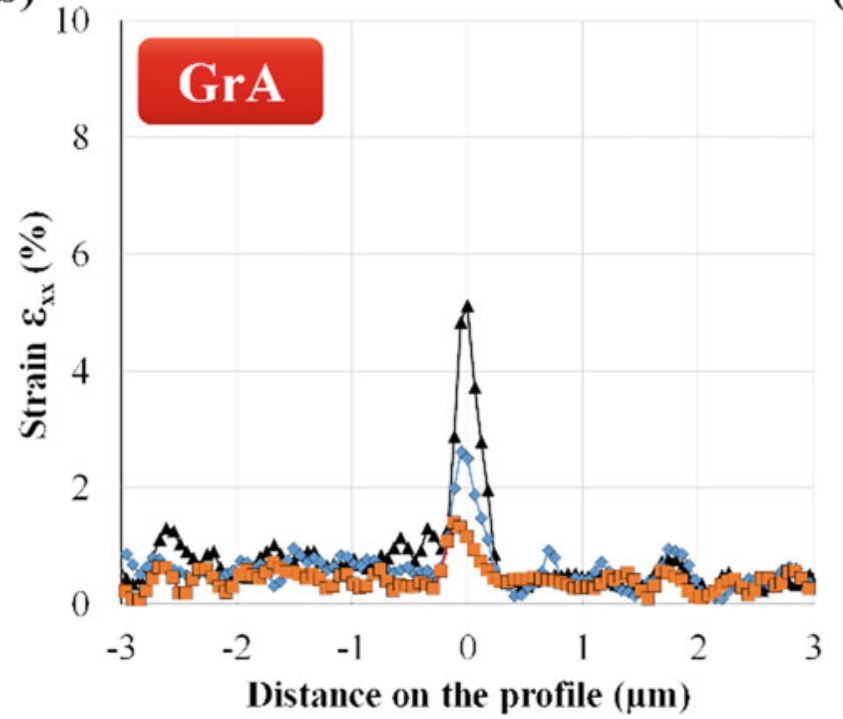

(c)

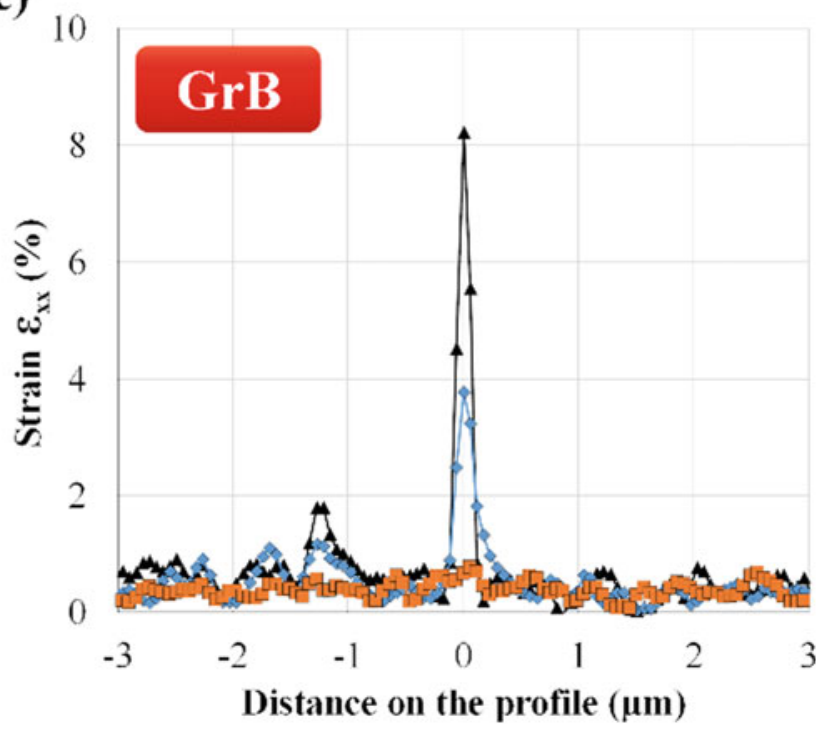

Fig. 4 Profile plot of the strain intensity across slip bands in GrA and GrB near and parallel to the NMI/matrix interface. a Illustration of both the profile lines in GrA and GrB in the strain and EBSD maps, profile plot of the strain intensity $\left(\varepsilon_{x x}\right)$ for the three first stress-strain conditions for $\mathbf{b} \mathrm{GrA}$, and $\mathbf{c} \mathrm{GrB}$ 
Table 1 Possible slip systems associated to the active planes in GrA and GrB, along with the Schmid factor along LD and Burgers vectors

\begin{tabular}{cccc}
\hline & \multicolumn{3}{c}{ GrA } \\
Slip system & $(\overline{1} 11)[01 \overline{1}]$ & $(\overline{1} 11)[101]$ & $(\overline{1} 11)[\overline{11} 0]$ \\
\hline$\mu$ & 0.433 & 0.427 & 0.006 \\
\hline $\overrightarrow{b_{\text {sample }}}$ & {$[1.070 .92 \overline{0.10}]$} & {$[\overline{0.05} \overline{0.91} 1.08]$} & {$[\overline{0.23} 1.380 .22]$} \\
\hline Slip system & $(1 \overline{1} 1)[110]$ & GrB & \\
\hline$\mu$ & 0.468 & $0.3 \overline{1} 1)[\overline{1} 01]$ & $(1 \overline{1} 1)[0 \overline{11}]$ \\
\hline$\underset{b_{\text {sample }}}{\longrightarrow} \overline{b_{\text {Gr B }}} / 2$ & {$[\overline{0.32} 1.38 \overline{0.07}]$} & {$[\overline{053} \overline{0.79} 1.04]$} & {$[\overline{0.45} \overline{0.18} \overline{1.33}]$} \\
\hline$\underset{\text { Gr A(act. }}{\longrightarrow}$ & 0.47 & -0.70 & -0.26 \\
\hline
\end{tabular}

Colored columns correspond to the system identified using HR-DIC and EBSD measurements

system in GrA. Damage after fatigue loading following slip activity in 718DA close and parallel to $\mathrm{NbC}$ and TiN was recently demonstrated by means of slip trace/crack shape 3D analyses and focused ion-beam cross-sections [4]. The morphology of monocrystalline NMIs is driven by its crystallographic growth. $\{001\}$ planes generally correspond to large facets in TiN and $\{111\}$ planes in NbC. Therefore, grains favorably oriented with NMIs facets were found prone to debonding at the NMI/matrix interface due to slip activity nearly parallel to the NMI/matrix interface trace at the surface of the specimen [4].

\section{Statistical Analysis of Microstructural Configurations Favoring or not NMI Cracking}

The analyzed ROIs exhibit different scenarios of slip events and NMI cracking under tensile loading. Different deformation/damage mechanisms were identified and extracted from these case studies.

NMI cracking and slip events within the metallic matrix far and near NMIs were investigated. As aforementioned, "grains near NMI" refers to "grains adjacent to NMI." The probability of slip events near and far from NMIs as well as NMI cracking was investigated for all the ROIs and all the loading conditions (Fig. 5). The probability of an event (POE) at a given strain level is defined by the sum of occurrence of the event for a given category, i.e., slip activity far from the NMI, slip activity within the grains adjacent to the NMI and NMI cracking, divided by the number of ROIs. For instance, if five ROIs exhibit slip activity within at least a grain adjacent to the NMI, the POE for "Slip act. neigh. grain" is $25 \%$ (5 ROIs over 20 ROIs in total). POE increased for the three events with the stress-strain increase. POE for slip rapidly saturates at $100 \%$ at $0.2 \%$ offset yield strength while POE for NMI cracking gradually increases. POE of NMI cracking is equal to 24 and $36 \%$ at 0.75 and $1.3 \%$ total strain, while all ROIs exhibit slip activity in grains adjacent to NMIs. For comparison, POE curves for cracked NMIs taken from Ref. [5] were added in Fig. 5. Owing to the higher yield strength of the 718DA in Ref. [5], the POE was plotted as a function of the macroscopic stress (blue dashed curve) and of the total strain (red unbroken curve). The good agreement between the present results and the POE representation as a function of the strain from Ref. [5] demonstrates the strain-controlled mechanism to crack NMIs. In other words, the sensitivity to crack NMI is directly correlated to the macroscopic yield strength of the Alloy 718. This point is crucial since it shows the damage density present after the first cycle in fatigue depending on the stress-strain amplitude. Despite a huge difference in stiffness between NMIs and the metallic matrix, the probability of slip within grains is higher far from the NMIs compared to the grains adjacent to NMIs at low stress-strain levels. This difference in POE could arise from the sampling method, taking into account more grains in the region far from NMIs.

A closer investigation in the vicinity of NMIs was conducted to evaluate the potential interaction between NMI cracking and slip activity within neighboring grains or at twin boundaries intercepting NMIs. Therefore, different categories were identified and reported in Table 2. Strain localization along twin boundaries intercepting NMIs was identified from merged maps of EBSD and $\varepsilon_{x x}$ strain fields. 
Fig. 5 Probability of slip events near and far from NMIs as well as NMI cracking, denoted as probability of event (POE) for all the loading conditions.

Comparison with POE from Ref. [5]

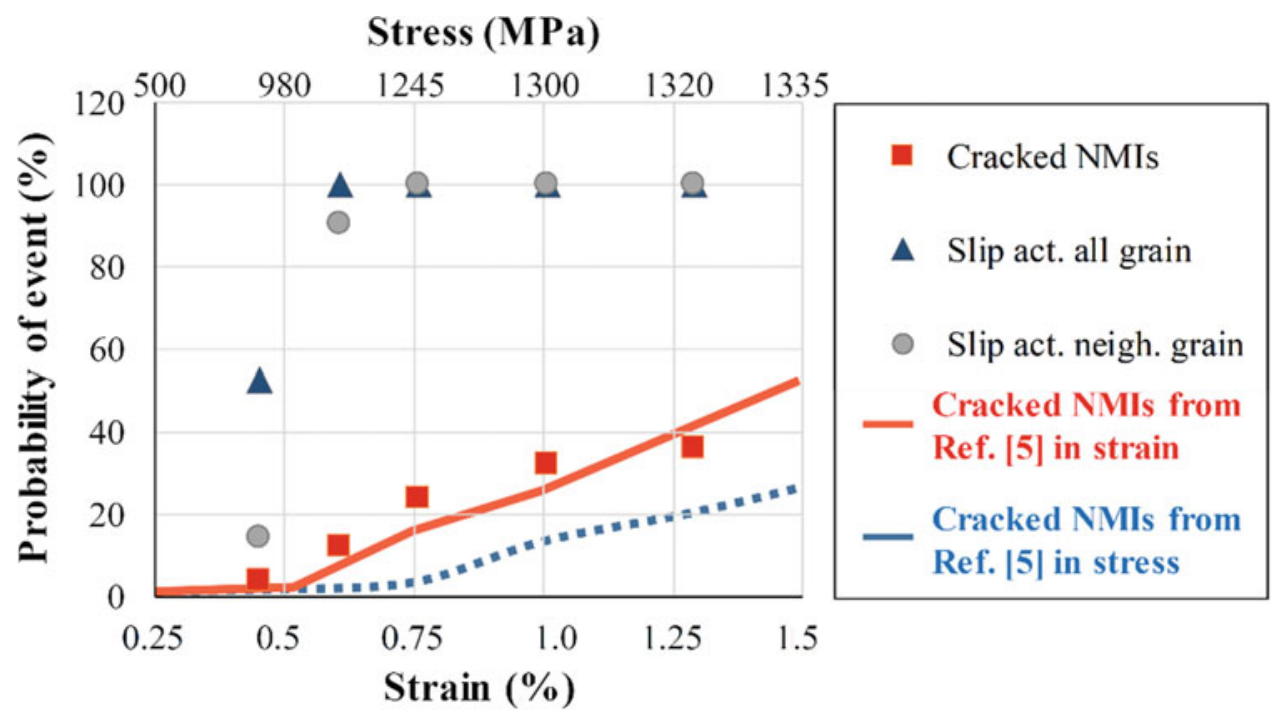

Table 2 Statistical analysis of possible interactions between slip activity and NMI cracking

\begin{tabular}{cccccccc}
\hline & \multicolumn{3}{c}{ NMI not cracking } & \multicolumn{3}{c}{ NMI cracking } \\
& TB/no slip & TB/slip & $\begin{array}{c}\text { Transg./ } \\
\text { slip }\end{array}$ & TB/slip & Transg./slip & $\begin{array}{c}\text { No slip } \\
\text { interaction }\end{array}$ \\
\hline Quantity (-) & 35 & 6 & 14 & 3 & 0 & 6 \\
\hline $\begin{array}{c}\text { Fraction per } \\
\text { category (\%) }\end{array}$ & 64 & 11 & 25 & 33 & 0 & 67 \\
\hline Fraction of TB (\%) & 79 & 14 & - & 7 & - & - \\
\hline
\end{tabular}

As long as no slip system was active along TB, NMI cracking did not occur. It also means that the elastic stress concentration on both sides of the TB is not sufficient to induce NMI cracking.

It is worth noting that $79 \%$ of TB intercepting NMIs were not found to develop slip activity. $14 \%$ of TBs developed slip but strain localization was not sufficient to crack the NMI. Only $7 \%$ of the TBs lead to slip-assisted NMI cracking. In addition, transgranular strain localization in contact with NMIs was also observed but never led to NMI cracking. This difference in slip-assisted NMI cracking could arise from the fact that the amplitude of the strain localization is more intense at TB compared to transgranular slip localization, as reported by Liu et al. [15].

Furthermore, most NMI cracking is associated with no slip interaction or no TB interaction. While slip-assisted NMI cracking was observed in the present study, most NMI cracking is due to strain incompatibility between NMIs and neighboring grains at the high end of the elastic regime without slip interaction.
In addition, the evolution of the slip localization from near and parallel to TB to transgranular sites was found with increasing stress-strain levels. This point is in good agreement with a previous study [15], showing this transition in slip events at nearly the $0.2 \%$ offset yield strength.

Finally, the majority of the NMIs did not necessarily crack even in the macroscopic plastic regime since $60 \%$ of the NMIs did not crack after $\varepsilon_{\text {tot }}=1.3 \%$, i.e., a plastic strain of $0.6 \%$. While not cracking, intense strain localization was observed surrounding NMIs.

It is worth mentioning that a large number of scenarios can occur depending on the NMIs (size, nature, orientation, clustering, etc.) and the neighboring grains (size, metallurgical state, orientation, etc.). Understanding microstructural features leading to severe strain localization or damage in the presence of NMIs is difficult due to the large number of scenarios. This partial conclusion is in good agreement with the numerical investigation conducted by Bandyopadhyay and Sangid [23] and Bergsmo and Dunne [22]. 


\section{Conclusions}

Elementary mechanisms of deformation in the vicinity or far from NMIs were investigated using HR-DIC and H-DIC after tensile loading. Slip-assisted NMI cracking was found to occur, especially when intense strain localization along a TB intercepts the NMI. Transgranular slip localization was not intense enough to crack NMIs under monotonic tensile loading. While slip activity is highly possible from $70 \%$ of the macroscopic yield strength (YS), few NMIs cracked under tensile loading. Within the NMIs that initiated cracks, $67 \%$ cracked below $90 \%$ of the macroscopic YS without the presence of slip activity in the neighboring grains. These deformation/damage mechanisms depend on the microstructural configurations surrounding the NMI, i.e., if TBs are intercepting the NMI or not, if grains are favorably oriented for transgranular slip intercepting the NMI, if only slip localization parallel to the NMI/matrix interface develops. These statistical analyses of slip/NMI interaction are highly important for further investigation under fatigue loading.

Acknowledgements The authors are grateful to Safran Aircraft Engines for providing materials and financial support. The authors are grateful to Jia H. Liu (ETS) and Florence Hamon (Pprime institute) for their help with mechanical testing and stimulating discussions.

\section{References}

1. Fayman YC (1987) Microstructural characterization and elemental partitioning in a direct-aged superalloy (DA 718). Mater Sci Eng 92:159-171. https://doi.org/10.1016/0025-5416(87)90166-2.

2. Krueger DD (1989) The development of direct age 718 for gas turbine engine disk applications. In: Superalloy 718: Metallurgy and Applications. Pittsburgh (USA), p 279-296.

3. Nicolay A, Franchet J-M, Bozzolo N, Cormier J (2020) Metallurgical analysis of direct aging effect on the tensile and creep properties in Inconel 718 forgings. In: Superalloys2020. Champion, PA (USA).

4. Texier D, Stinville J-C, Echlin MP, et al (2019) Short crack propagation from cracked non-metallic inclusions in a Ni-based polycrystalline superalloy. Acta Mater 165:241-258. https://doi. org/10.1016/j.actamat.2018.11.051.

5. Texier D, Casanova-Gómez A, Pierret S, et al (2016) Microstructural features controlling the variability in low-cycle fatigue properties of alloy Inconel 718DA at antermediate Temperature. Metall Mater Trans A Phys Metall Mater Sci 47:1096-1109. https://doi.org/10.1007/s11661-015-3291-8.

6. Texier D, Cormier J, Villechaise P, et al (2016) Crack initiation sensitivity of wrought direct aged alloy 718 in the very high cycle fatigue regime: the role of non-metallic inclusions. Mater Sci Eng A 678:122-136. https://doi.org/10.1016/j.msea.2016.09.098.

7. Späth N, Zerrouki V, Poubanne P, Guedou J-Y (2001) 718 superalloy forging simulation: a way to improve process and material. In: Superalloys 718, 625, 706 and various derivatives. Pittsburgh (USA), p 173-183.
8. Alexandre F, Deyber S, Pineau A (2004) Modelling the optimum grain size on the low cycle fatigue life of a $\mathrm{Ni}$ based superalloy in the presence of two possible crack initiation sites. Scr Mater 50:25-30. https://doi.org/10.1016/j.scriptamat.2003.09.043.

9. Deng G-J, Tu S-T, Zhang X-C, et al (2015) Grain size effect on the small fatigue crack initiation and growth mechanisms of nickel-based superalloy GH4169. Eng Fract Mech 134:433-450. https://doi.org/10.1016/j.engfracmech.2015.01.002.

10. Miao J, Pollock TM, Wayne Jones J (2009) Crystallographic fatigue crack initiation in nickel-based superalloy René 88DT at elevated temperature. Acta Mater 57:5964-5974. https://doi.org/ 10.1016/j.actamat.2009.08.022.

11. Miao J, Pollock TM, Wayne Jones J (2012) Microstructural extremes and the transition from fatigue crack initiation to small crack growth in a polycrystalline nickel-base superalloy. Acta Mater 60:2840-2854. https://doi.org/10.1016/j.actamat.2012.01.049.

12. Stinville J-C, Vanderesse N, Bridier F, et al (2015) High resolution mapping of strain localization near twin boundaries in a nickel-based superalloy. Acta Mater 98:29-42. https://doi.org/10. 1016/j.actamat.2015.07.016.

13. Stinville J-C, Lenthe WC, Echlin MP, et al (2017) Microstructural statistics for fatigue crack initiation in polycrystalline nickel-base superalloys. Int J Fract 208:221-240. https://doi.org/10.1007/ s10704-017-0241-z.

14. Stinville J-C, Martin E, Karadge M, et al (2018) Competing modes for crack initiation from non-metallic inclusions and intrinsic microstructural features during fatigue in a polycrystalline nickel-based superalloy. Metall Mater Trans A. https://doi.org/ 10.1007/s11661-018-4780-3.

15. Liu JH, Vanderesse N, Stinville J-C, et al (2019) In-plane and out-of-plane deformation at the sub-grain scale in polycrystalline materials assessed by confocal microscopy. Acta Mater 169:260 274. https://doi.org/10.1016/j.actamat.2019.03.001.

16. Haldipur $P$ (2006) Material characterization of nickel-based superalloys through ultrasonic inspection. Ph.D. thesis, Iowa State University (USA).

17. Stinville J-C, Callahan PG, Charpagne M-A, et al (2020) Direct measurements of slip irreversibility in a nickel-based superalloy using high resolution digital image correlation. Acta Mater 186:172-189. https://doi.org/10.1016/j.actamat.2019.12.009.

18. Shenoy MM, Kumar RS, McDowell DL (2005) Modeling effects of nonmetallic inclusions on LCF in DS nickel-base superalloys. Int J Fatigue 27:113-127. https://doi.org/10.1016/j.ijfatigue.2004.06.008.

19. Zhang J, Prasannavenkatesan R, Shenoy MM, McDowell DL (2009) Modeling fatigue crack nucleation at primary inclusions in carburized and shot-peened martensitic steel. Eng Fract Mech 76:315-334. https://doi.org/10.1016/j.engfracmech.2008.10.011.

20. Prasannavenkatesan R, Zhang J, McDowell DL, et al (2009) 3D modeling of subsurface fatigue crack nucleation potency of primary inclusions in heat treated and shot peened martensitic gear steels. Int J Fatigue 31:1176-1189. https://doi.org/10.1016/j. ijfatigue.2008.12.001.

21. Prasannavenkatesan R, Przybyla CP, Salajegheh N, McDowell DL (2011) Simulated extreme value fatigue sensitivity to inclusions and pores in martensitic gear steels. Eng Fract Mech 78:1140 1155. https://doi.org/10.1016/j.engfracmech.2011.01.027.

22. Bergsmo A, Dunne FPE (2020) Competing mechanisms of particle fracture, decohesion and slip-driven fatigue crack nucleation in a PM nickel superalloy. Int J Fatigue 135:105573. https://doi.org/10. 1016/j.ijfatigue.2020.105573.

23. Bandyopadhyay R, Sangid MD (2019) Crystal plasticity assessment of inclusion- and matrix-driven competing failure modes in a nickel-base superalloy. Acta Mater 177:20-34. https://doi.org/10. 1016/j.actamat.2019.07.024. 
24. Kartal ME, Dunne FPE, Wilkinson AJ (2012) Determination of the complete microscale residual stress tensor at a subsurface carbide particle in a single-crystal superalloy from free-surface EBSD. Acta Mater 60:5300-5310. https://doi.org/10.1016/j.actamat.2012. 06.027.

25. Kammers AD, Daly S (2011) Small-scale patterning methods for digital image correlation under scanning electron microscopy. Meas Sci Technol 22:1-12. https://doi.org/10.1088/0957-0233/22/ $12 / 125501$.

26. Kammers AD, Daly S (2013) Digital image correlation under scanning electron microscopy: methodology and validation. Exp Mech 53:1743-1761. https://doi.org/10.1007/s11340-013-9782-x.

27. Stinville J-C, Echlin MP, Texier D, et al (2016) Sub-grain scale digital image correlation by electron microscopy for polycrystalline materials during elastic and plastic deformation. Exp Mech 56:197-216. https://doi.org/10.1007/s11340-015-0083-4
28. Valle V, Hedan S, Cosenza P, et al (2015) Digital image correlation development for the study of materials including multiple crossing cracks. Exp Mech 55:379-391. https://doi.org/ 10.1007/s11340-014-9948-1.

29. Bourdin F, Stinville JC, Echlin MP, et al (2018) Measurements of plastic localization by heaviside-digital image correlation. Acta Mater 157:307-325. https://doi.org/10.1016/j.actamat.2018. 07.013.

30. Rathod N, Gupta SD, Gupta SK, Jha PK (2011) First-principles study of structural, electronic, elastic, phonon, and thermodynamical properties of the niobium carbide. Solid State Phenom 171:6777. https://doi.org/10.4028/www.scientific.net/ssp.171.67.

31. Kim JO, Achenbach JD, Mirkarimi PB, et al (1992) Elastic constants of single-crystal by line-focus acoustic microscopy nitride films measured. J Appl Phys 72:1805-1811. https://doi.org/ $10.1063 / 1.351651$. 